\title{
The role of cell-free circulating microRNA in diagnostics in patients with rheumatoid arthritis
}

\author{
Agnieszka Paradowska-Gorycka, Barbara Stypińska
}

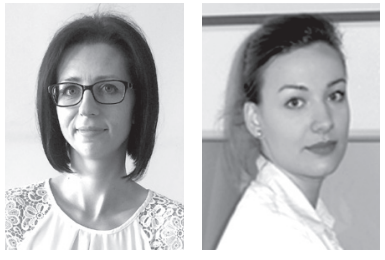

Department of Biochemistry and Molecular Biology, National Institute of Geriatrics, Rheumatology and Rehabilitation, Warsaw, Poland

Within the past years, more and more attention has been devoted to epigenetic anomalies which regulate gene expression without altering the DNA sequence. Therefore, epigenetic modifications are emerging as key pathogenic features of immune diseases, including rheumatoid arthritis (RA), and provide an additional window for understanding the possible mechanisms involved in the pathogenesis of disease [1, 2]. DNA methylation, histone modification, nucleosome positioning and microRNAs (miRNAs) are major mechanisms of epigenetic gene regulation. These epigenetic modifications modulate chromatin architecture, thereby participating in the regulation of gene transcription and final interpretation of the encoded genetic information.

Alterations in their profile influence the activity of the immune system.

Rheumatoid arthritis is a systemic, inflammatory/ autoimmune, polygenic disease affecting millions of people worldwide. Its etiopathology is attributed to a crosstalk between genetic predisposition and environmental factors $[1,3]$. This heterogeneous disorder is characterized by chronic synovitis and a fluctuating clinical course that may result in long-term disability and reduced quality of life in many patients [1, 2, 4]. Although a large panel of effective biotherapies is now available to the rheumatologist, the most important challenging issue remains to find parameters/biomarkers for early diagnosis, monitoring disease activity and response to therapy, and finally understanding RA pathogenesis $[1,3]$. Among molecules that are able to fulfill this requirement, microRNAs (miRNAs; miRs) represent an ideal, highly specify and sensitive, noninvasive class of blood-based biomarkers, as they can be aberrantly expressed even in the different stages of RA progression, and expression patterns of miRNAs in human rheumatic diseases appear to be tissue-specific [4].

MiRNAs represent a large family of small, evolutionarily conserved endogenous noncoding RNAs, which are integral parts of gene expression networks that determine cell identity and function. MiRNAs modulate protein expression through either repression of translation or by inducing mRNA degradation and turnover, and their signatures have become one of the most fascinating interests in current biology and medical science [2]. Human miRNA genes are located on all chromosomes except chromosome $\mathrm{Y}$, are mainly observed in introns (70\%), constitute only $3 \%$ of the human genome, and regulate about $90 \%$ of different genes $[3,5]$. These molecules participate in regulation of the expression of genes involved in the fundamental biological processes related to development, proliferation and apoptosis as well as pathological processes including autoimmunity $[1,6]$. It has been shown that miRNAs modulate $T$ cell selection and affect the differentiation and effector/regulatory functions of $\mathrm{T}$ and $\mathrm{B}$ cells, thereby modulating autoimmune pathogenesis [1]. Although miRNAs have an important role in defining and maintaining the gene expression involved in Th17 cell differentiation, they also participate in maintenance of self-tolerance regulating Treg cell function.

The first evidence that miRNAs play an important role in RA was first reported less than a decade ago [2, 5]. Although several miRNAs (miR-146, miR-155, miR-23, miR-125, miR-181, miR-223) act as relevant regulators of innate and adaptive immune cell development, and they have been found to contribute to RA pathogenesis, unique miRNA signatures in RA have not been found yet [7]. The discovery of miRNA is one of the major recent scientific breakthroughs and has revolutionized knowledge of gene regulation. An increasing number of studies have demonstrated that dysregulation of miRNAs in peripheral blood mononuclear cells, isolated $T$ cells, synovial tissues and synovial fibroblasts contributes to inflammation, degradation of extracellular matrix and invasive behavior of resident cells [1]. Tissue miRNAs act not only as key molecules in intracellular regulatory net- 
works for gene expression, but also as biomarkers for several pathological conditions [8]. Recent studies have suggested that circulating miRNAs may be suitable for clinical use as they are present in a stable, cell-free form in body fluids such as plasma or serum that are protected from endogenous RNase activity [8, 9]. Serum/plasma miRNAs are stable at room temperature for up to 24 hours and resistant to freeze-thawing from $-80^{\circ} \mathrm{C}$ to room temperature up to eight times [8]. Recent evidence has suggested that the levels of miRNAs in serum/plasma are frequently higher compared to synovial fluid. Furthermore, the synovial fluid miRNAs were similar to miRNAs generated by synovial tissue. These facts suggest that synovial tissue and infiltrating cells are a major source of synovial fluid miRNAs, whereas miRNAs present in serum/plasma are secreted by various tissues [8]. Moreover, because rheumatoid arthritis is a systemic disease in which not only joints but also other organs may be affected, a cell-free circulating miRNA signature in serum/plasma is of great interest [3].

However, the investigation of the role of miRNA dysregulation in $\mathrm{RA}$ is not so simple. First, rheumatoid arthritis is a multifactorial disease with several sets of clinical manifestations in each individual. Second, a single miRNA regulates multiple genes, and multiple miRNAs may regulate a single mRNA [3]. Finally, the usefulness of miRNAs as clinical biomarkers may depend on several pre-analytic factors during the sample collection, RNA isolation and qRT-PCR analysis as well as on the use of appropriate references for data analysis [10].

Collectively, the understanding of the role of miRNAs in rheumatoid arthritis pathogenesis as well as identification, characterization, and modulation of specific miRNA expression in RA is of great interest and opens a new way to discover not only novel molecular diagnostic markers but also new gene therapy strategies for targeting RA.

The authors declare no conflict of interest.

\section{References}

1. Castro-Villegas C, Perez-Sanchez C, Escudero A, et al. Circulating miRNAs as potential biomarkers of therapy effectiveness in rheumatoid arthritis patients treated with anti-TNF. Arthritis Res Ther 2015; 17: 49.

2. Vicente R, Noel D, Pers M, et al. Deregulation and therapeutic potential of microRNAs in arthritic diseases. Nat Rev 2016; 12: 211-220.

3. Duroux-Richard I, Jorgensen C, Apparailly F. What do microRNAs mean for rheumatoid arthritis? Arthritis Rheum 2012; 64: 1-20.

4. Filkova M, Aradi B, Senolt L, et al. Association of circulating miR-223 and miR-16 with disease activity in patients with early rheumatoid arthritis. Ann Rheum Dis 2014; 73: 1898-1904.
5. Singh RP, Massachi I, Manickavel S, et al. The role of miRNA in inflammation and autoimmunity. Autoimmun Rev 2013; 12: 1160-1165.

6. Smigielska-Czapiel K, van der berg A, Jellema P, et al. Comperashive analysis of miRNA expression in T-cell subsets of rheumatoid arthritis patients reveals defined signatures of naive and memory Tregs. Genes Immun 2014; 15: 115-125.

7. Chen XM, Huang QC, Yang SL, et al. Role of microRNAs in the pathogenesis of rheumatoid arthritis. Medicine 2015; 94 : e1326.

8. Murata K, Yoshitomi H, Tanida S, et al. Plasma and synovial fluid microRNAs as potential biomarkers of rheumatoid arthritis and osteoarthritis. Arthritis Res Ther 2010; 12: R86.

9. Mitchell PS, Parkin RK, Kroh EM, et al. Circulating microRNAs as stable blood based markers for cancer detection. PNAS 2008; 105: 10513-10518.

10. Churov AV, Oleinik EK, Knip M. MircoRNAs in rheumatoid arthritis: altered expression and diagnostic potential. Autoimmun Rev 2015; 14: 1029-1037. 